\title{
Knowledge Visualization and Business Analysis: Meaning as Media
}

\author{
Stefan Bertschi \\ University of Zurich \& loginb.com \\ stefan.bertschi@loginb.com
}

\begin{abstract}
This paper is the third in a series of papers designed to explore how "metaphorical meaning" can be used to support knowledge visualization. Within the present discussion the following is assumed: To understand the process of mind and organizational performance a view on meaningful expression is needed. In order to approach this collaborative task, knowledge visualization is seen as "business analysis" and the concepts of metaphor and media are seen as a carrier of meaning. This is meant to provide a better understanding of the visualization of knowledge for necessary alignment in business analysis.
\end{abstract}

\section{Introduction}

"Everything that is visible hides something else that is visible." René Magritte [1, p. 64]

This assumed paradox lies at the heart of a series of three papers which comes to an end with the present paper. As will become clear, I am far from reaching a final conclusion. However, by introducing a new comparative application of knowledge visualization, i.e. business analysis, the third paper opens the discussion in a new direction.

The first paper examined the metaphorical face of knowledge visualization and discussed the interrelation of so-called "linguistic learning" and mediation of knowledge through visualization [cf. 2]. According to this approach, knowledge is constantly being reconstructed through communication and the use of existing linguistic structures and patterns of learning. Hence, knowledge visualization aims at augmenting communication via graphic means which mediate knowledge through metaphors. The second paper discussed whether metaphor, meaning and perception are sufficiently dealt with in knowledge visualization [cf. 3]. Following the constructivist stance developed in the first paper, I introduced a deconstructivist approach to reveal the construction and reconstruction of meaning in knowledge transfer.

The present discussion starts where the previous papers ended: with the conclusion that knowledge visualizations "aim particularly at portraying the reconstruction of patterns" [2, p. 384] and with the insight that "collaboration is important for social negotiation on meaning, for the testing of ideas, and the exchange and discussion of different perspectives." Anyone who wants to "transfer knowledge to one or more individuals, with similar or different backgrounds" [4, p. 31], has to be aware that visualizations need to be shaped and customized "in a way that the recipient can reconstruct knowledge as intended by the sender" [4, p. 54]. Therefore, I start with a first thesis: To understand the process of internal competence (mind) and external performance (organizational, social) a view on meaningful expression is needed.

In the following sections I discuss perception, metaphor, business analysis and media, each in the context of meaning. Finally, I present some preliminary conclusions and an outlook that can be drawn from this discussion.

\section{Meaning and perception}

At present, I aim to extend the discussion on human perception and the feasibility of transfer intended in knowledge visualization (presented at the "Symposium on Knowledge and Argument Visualization" in 2007), rejoin the debate on metaphor and introduce a connection between visualization and business analysis. The current approach centers on the "process of people collectively using $[\ldots]$ assumptions in [perceiving and; S.B.] interpreting each other's actions" [5, p. 36]. Whereas individuals carry private representations, only mediating structures (or symbols which connect individuals) allow for private representations being aligned among individuals. It is significant that perceptions and subsequent private representations are constructed and reconstructed within the singular realms of individuals or, with other words, in their minds [cf. 5, p. 42]. 
Rubin's vase is an outstanding example for perception. Even though this figure-ground illustration is much older, it was Danish psychologist Edgar Rubin who made the "vase/profile illusion" famous in 1915 (see Figure 1). What is since known as "Rubin's vase" offers a mental choice of two equally valid interpretations: "we can alter our perception by directing our attention to either vase or face" [6, p. 154; cf. 7]. Attention can select either object as the "figure", thereby fundamentally distort perception.

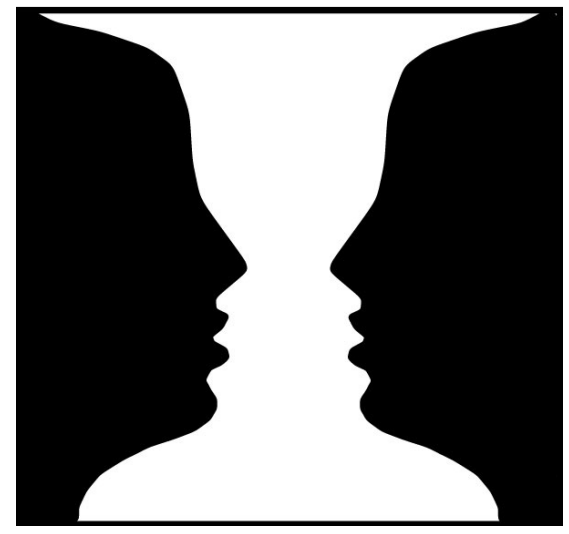

Figure 1 Rubin's vase

The preferred identification of objects is connected with the ability to perceive at will and to "differentiate" between "figure" and "background". In an experiment focused on these selection processes, it was argued whether one chooses those attributes of an object which are immediately relevant to the task in hand [cf. 6, p. 155]. It seems quite reasonable that task-relevant or meaningful attributes are selected at first. A second example is given in Akiro Kurosawa's film "Rashomon" (1950): A crime, involving rape and murder, is reported by four witnesses, each from their own point of view [cf. 8, p. 93], and each telling a completely different story. Giving four viewpoints of the incident, the movie leads to questions like: Which witness is telling the truth? What is the right perception? How can one tell?

These "multiple perceptions" lead to a second thesis: To align individual representations a "flow of significance" is needed. In order to understand the symbols that allow individuals to be linked [cf. 5, p. 36], underlying processes require the use of linguistic learning. One learns the meaning of a symbol by finding out "what it is" and then by creating a version of that symbols' meaning [5, p. 43]. Cultural representations are perceived and comprehended by exposure to the mediating structure and by applying meaning to this structure [cf. 5, p. 47]. This leads on to the argument that functional perception is dependent on the intended meaning of visualization [cf. 3, p. 342]; whilst meaning "is central to understanding human behavior." [9, p. xii] In the communication process our aim is to understand both sender and recipient, or even more, to connect them properly and align their understanding. However, consensus is not guaranteed, not even alignment is ensured. It is simply known that perception differentiates according to significance and meaning. Therefore, to constitute a meaningful practice within the transfer of knowledge, the interdependence of meaning and perception has to be acknowledged and understood.

In the previous paper I argued that the reasonable approach to evaluate the mechanisms and effectiveness of knowledge visualization has to be a type of reading "texts" (of all sorts) and is meant to consider, reveal and, finally, understand the underlying circumstances and contexts in which they are produced and received [cf. 3, p. 343]. This "understanding" was modeled on a process of learning [cf. 2; 18, p. 455]. Hence, there are sender and recipient, and the attempt to reveal how to analyze "meaning" in this mediating structure. For this purpose I use a diagram from Claude E. Shannon and Warren Weaver's "Mathematical Theory of Communication" (see Figure 2). In this depiction, "noise" is certain distortions and errors due to the transmission. Because of noise, "the received message exhibits [...] an increased uncertainty." In their aim to solve the technical problem of transmission and to design the most accurate communications channel, Shannon and Weaver considered noise to be "undesirable uncertainty." [10, p. 19] Additionally, noise is important for the semantic problem and the effectiveness problem of communication [cf. 10, p. 4]; both the contents and the quality of transmission are affected by noise.

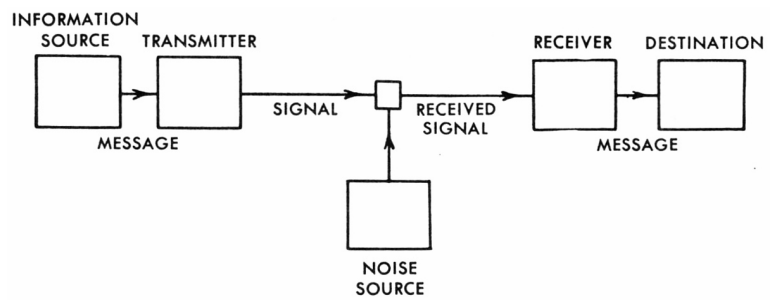

Figure 2 General communication system

Moving on from Shannon and Weaver's depiction of a general communication system, one can tentatively illustrate the relationship of the resulting "two" meanings (see Figure 3). Knowledge has to be reconstructed by the recipient; because the recipient has to accord meaning to the incoming message, he or she is not at all passive, "but participates in the structuring of external reality." [3, p. 345] Therefore, the recipient is also the interpreter and confronted with "new" noise. 
This noise is now to be found on the right side of the receiver. It is worthwhile noting that Shannon and Weaver's original noise is still included in the "visualization" which takes place between sender and receiver. In this two-part process, i.e. presentation by the sender and subsequent separate formation of representations by the recipient, the problem of "noise" and "meaning" is encountered as a problem of two noises and two meanings.

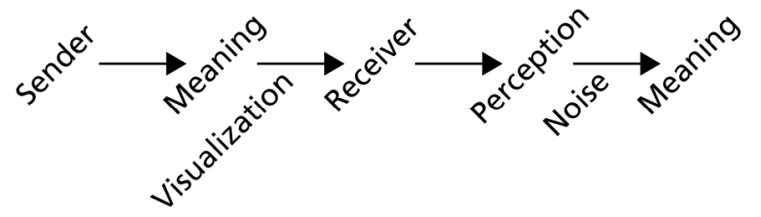

Figure 3 Two meanings

The analysis of this communication process should address the "how" (how does it happen?), which in a common understanding leads to the "why" (why does it happen?), instead of the "what" (what happens?), and a deconstructivist approach has proven to be a suitable tool for addressing construction [cf. 3, p. 343]. But it is the "why" which has to direct our approach to the "how" of the mediating structure and perception [cf. 5, p. 44]. The structure linking sender and recipient could be the metaphor (as indicated in [2] and [3]), at the same time providing an answer on the "how" of the mediating structure (i.e. its functioning), and on how to address the problem described. This means in short and wrapped in a third thesis: Metaphor is typified language use and a means to simplify the distinction of noises and meanings.

\section{Meaning and metaphor}

Metaphors may establish conventional meanings and work as "instructions" for agreement [cf. 5, p. 42]. They provide previous constructions and evoke a connected mental picture which, in the best of cases, is identical or at least significantly approximated in sender and recipient. At the same time, metaphors "admit endless or at least open-ended interpretations"; what they mean or communicate is never fixed in advance [11, p. 205; cf. 12]. Even metaphors cannot ensure that meaning is transferred identically to all recipients. This is illustrated in the following example: In Nigeria people use the metaphor "She is my bedbug" as a term of affection for their lover because bedbugs are considered cute. Given our, say Western European presuppositions about bedbugs, the same metaphor would express something very different: saying that he or she is a nuisance [cf. 11, p. 208]. This example shows same content that can have different metaphorical meanings in different contexts, again distorting perception. However, the conception of metaphor as the "fountain of meaning and truth" plays a foundational role in human understanding [cf. 3, p. 344]; it plays a central role in the way we make sense of the world. Because "there is no accepted understanding about the way metaphors work" [13, p. 393], I understand metaphors to be a capability to comprehend and shape experience, "like seeing or touching or hearing, with metaphors providing the only way to perceive and experience much of the world" [14, p. 239]. It is understood that "metaphor [...] results in an active and unified apprehension of knowledge." [13, p. 394] Metaphors organize knowledge.

As a return to the previous papers, I subscribe to images of organizations (and organizing) adhering to a linguistic model (hence, linguistic learning in [2]) or, in other words, "language-based conceptualizations of organizations" [13, p. 395, 401]. It will soon become clear why "organization" is introduced as a central aspect of the present paper. In this logic, knowledge visualization contributes to the analysis of meaning in communication. With its intangible connection to metaphor [cf. 4; 16], "the essence of [...] [knowledge visualization] is understanding and experiencing one kind of thing in terms of another" $[14$, p. 5]. By visualizing complex issues, these processes of understanding and experiencing are embedded in the way we act, interact and - again - make sense of the world.

In regard to organizations a plausible example is found in the metaphor "Work is a game." In this metaphor, work is seen as a game, including players, moves and strategies. Thereby, metaphors "help to structure beliefs and guide behavior in organizations, express abstract ideas, convey vivid images that orient our perceptions and conceptualizations, transfer information, legitimate actions, set goals, and structure coherent systems" [13, p. 396]. Furthermore, they can be used to highlight and reproduce practices and patterns, perceptions and reactions as well as norms, motives and meanings.

\section{Meaning and business analysis}

Seeing meaning both as part of one's mind and as a social construction is an aspect that is also contained in understanding "business analysis". According to this approach, differences in meaning and difficulties in understanding have to be overcome or analysis and visualization will inevitably fail. This holds especially true for the two meanings involved in the transfer of knowledge (see Figure 3). They emerge because the corresponding qualities are "constructed rather than deciphered" [15, p. 751], resulting in the fact that meaning is emergent and individual cognitive 
processes differ immensely. In order to manage and transfer knowledge, these processes of construction and reconstruction need to be analyzed and understood.

Originally, knowledge visualization was set as a "new field [...] in the context of business knowledge management." [4, p. 52] I believe that this focus is too restrictive, as there is more potential in the construction and reconstruction of knowledge within a wider understanding of organization and collaboration. To reflect a change for IV'09, the "Symposium on Knowledge and Argument Visualization" has been renamed to "Knowledge Visualization and Visual Thinking". For the intended connection with business analysis, I extend knowledge visualization beyond the mere business application and relate it to general organizational realms and meaningful thinking about these realms. As will become clear, the same applies to my understanding of business analysis.

\subsection{Business analysis}

Business analysis is commonly understood as an analytical and consultative position, liaising between the business side and service provision in organizations. It combines the analysis of customer requirements, the questioning of existing and prospective processes, the structuring of and advice on change and development; but business analysis is also concerned with managing relationships and transferring knowledge within an organization. An even narrower definition limits business analysis to the smooth integration of new technologies and business processes, translating business requirements into IT requests.

The International Institute of Business Analysis (IIBA), the leading professional association, defined its field as follows: "Business Analysis is the set of tasks, knowledge, and techniques required to identify business needs and determine solutions to business problems. Solutions often include a systems development component, but may also consist of process improvement or organizational change." [17, p. 12] Likewise, their definition of the role of business analyst reads: "A business analyst works as a liaison among stakeholders in order to elicit, analyze, communicate and validate requirements for changes to business processes, policies and information systems. The business analyst understands business problems and opportunities in the context of the requirements and recommends solutions that enable the organization to achieve its goals." [17, p. 13, my italics] Determining solutions to organizational problems and identifying needs of stakeholders are at the core of this profession. The business analyst acts as a bridge between the involved parties, translating different views into one.
Business analysis as it is understood here is the "management of meaning" which is used "when communicating organizational change to inform and inspire", to "facilitate knowledge production" and to "create shared experiences" [13, p. 405]. The process of analyzing and steering has to consider how identities are created (minds, roles, images etc) and how meaning is framed within processes (stories, slogans, artifacts etc). A practical example is the construction of vision and mission statements. It is crucial to understand that this cannot be a one-way process: organizational members are "shaping and being shaped in their everyday actions, interactions, and relationships" [13, p. 407, my italics]. Conversation and collaboration are paramount; and these mediated exchanges of different perspectives facilitate the connection with business analysis because "metaphor is seeing something from the viewpoint of something else" [16, p. 170, my italics], e.g. seeing technology/change from the viewpoint of business/organization and vice versa.

It is an outstanding capability of metaphors "to create previously unapprehended relationships." [13, p. 403, my italics] The metaphor's relation to meaning allows us to relate both metaphor and meaning to general connections and collaborations, as included in my understanding of business analysis. Wherever a common understanding is sought, there is a need for aligned meaning. Because sender and recipient have their independent minds, it has to be taken into account that "meaning exists outside discursive reproduction" $[13$, p. 404]. Hence, one is thrown back to the problem of at least two meanings.

\subsection{Context}

It does not come as a surprise that the envisioned alignment depends on the context in which one is supposed to analyze, interpret and execute it. Business analysis, like knowledge visualization, depends on awareness, perception and the recognition of meaning [cf. 3, p. 345]. How is something done and why? Is it able to fulfill its objectives? In both fields it is important to understand that the receiver is not a passive recipient of information but participates in the structuring of knowledge; each recipient has his or her own mindset. These mind-sets involve complex subjective constructions which are constantly being modified in communication. Knowledge visualization already demonstrated awareness for the interpretational process of meaning [cf. 4, p. 121], and the same should apply to business analysis. Whereas the understanding of needs for change is central in business analysis, communication and delivery are equally important. Without understanding the meaning of these processes, their communication is restricted, particularly if the need for 
aligning different views prevails. Loaded with the task of collaborative meaning management, choosing the right mode of communication is essential to get appropriate information, to save time and money, to avoid confusion and to obtain support from stakeholders.

Both knowledge visualization and business analysis are about constructing and reconstructing (contextual) meaning. Business analysts would have to inquire in which situations and how they can apply and benefit from knowledge visualization: How can visualization and visual metaphors be used to improve their liaison practice? Which issues and risks may emerge in this context? What methods exist or need to be developed? In this regard, business analysis would become a field of application for knowledge visualization and a thorough discussion is needed. At present, I focus on mediated meaning as its precondition.

It is the "seeing something from another viewpoint" knowledge visualization and business analysis have in common. This leads to a fourth thesis: Both knowledge visualization and business analysis address the question how different people are able to perceive the same organizational "world" (i.e. knowledge) at the same time. "[M]aking sense of ambiguous and complex situations through conversations and dialogue" becomes increasingly important $[19$, p. 5]. On this note, the business analyst is situated "in between" the heads (mind, individual, sense, meaning) and the outside (environment, organization, situation, meaning). The same applies to knowledge visualization which "aims to improve the transfer and the creation of knowledge" by giving people "richer means to express what they know" [4, p. 135, my italics]. These richer means refer to a mediating process involving visualization; they refer to means of communication and collaboration in the context of meaning.

\section{Meaning and media}

The approach proposed here reflects the problem of transfer as related to a "medium" or "carrier" of meaning [cf. 3, p. 343]. Metaphors act as carriers or "transporters of meaning" [9, p. 225]. Carriers facilitate a common understanding because they are already situated in context [cf. 9, p. 232]. For instance, I have an understanding of what " $\mathrm{X}$ " means to me personally because someone was able to (visually) demonstrate why. Hence, this approach is not merely based on what is said and how it is said, but on why it is said and why visualizations mean something. Such a view is pivotal to understanding the subjective and contextual meaning of people's experiences and to differentiate them from noise.

In regard to aforementioned collaboration, it is particularly important to understand meaning [cf. 2, p.
385]. These meanings have to be reflected in a "medium", some kind of carrier or process interlinking different views. Again, it leads to the question how these meanings can be expressed and transferred efficiently and effectively. Thinking of richer means to express knowledge, most prominently digital interactive media and Adobe Flash come to mind. As a technology that integrates audio, video and high-resolution graphics, the defining characteristic of so-called "rich media" is the following: it features dynamic motion which can occur over time or as a direct response to interaction. (The present paper merely points to such media for demonstration, e.g. Adobe Flex or the more visually rich ILOG Elixir, including 2D and 3D charts, treemaps, map-based dashboards, organization charts, gauges and Gantt charts.)

Microsoft PowerPoint, the standard tool for knowledge visualization, already "integrates visual media into an overall structure of speech", even though the process of presentation is only "half-automated" and relates on its "live" performance [20, p. 89]. This situation reminds of the business analyst, the collaborative approach and its dependent performance of meaning. Furthermore, the positive aspect of mediation technologies lies in the "dissolution of institutional boundaries of knowledge" [20, p. 92]. Like in other computermediated communication, content (i.e. knowledge) does not affect form. This versatility demands a "recontextualization" which in turn allows addressing the problem of the two meanings.

Moving pictures, however, retain their context and thereby constitute meaning, not the least because they are the most common way one experiences the world. With other words, "writing a caption under a picture is not the only way to disambiguate it; turning a static picture into an animated one might solve the problem, too." [21] Rich media might be such a solution: providing richer means to collaborate globally, leading to meaningful information and facilitating an aligned understanding. Using the same data or information, in an ideal future, "the [rich media] application [intuitively; S.B.] adapts to the uses of that data, depending on who is using the application." [22, p. 5] However, the versatility (multiple contexts, meanings etc) remains a problem which has to be accounted for. Business processes and their wider organizational variants are equally affected and relate these mediating structures to analysis and visualization. Finally, it is this "knowledge transmission" [20, p. 91] which leads back to Magritte's "hidden visible" and to the beginning of the paper.

\section{Conclusion}

The envisioned alliance of knowledge visualization and mediated business analysis may not only have the 
potential to solve "the predominant problems in organizations", i.e. to communicate complex contents to diverse audiences [4, p. 131], but to address problems related to this transfer. Starting with perception and meaning, I discussed the relevance of existing and new components: metaphor, business analysis and media. I underlined the need for a view on meaningful expression and the "flow of significance" in order to understand the process of mind and organizational performance, and to link these individual representations (or minds). I reiterated the importance of metaphor as a means to simplify the distinction of noises and meanings, and I combined knowledge visualization and business analysis to address how and why different people perceive the same meaning.

The process of construction and reconstruction has to take place in a mediating structure, as a mediating process. The resulting view on "rich media" originates in the assumption that visualizations will lose their character as static objects in a familiar sender-recipient setting. Instead, they will increasingly "establish an iterative, collaborative process where the visualization (and new knowledge) is dynamically co-created." [4, p. 140] Knowledge transmission, as the main conclusion to this series of papers, means construction and reconstruction of meaning. The proposition of the present discussion is "to understand" and more literally to "comprehend the meaning of something" [18, p. 452]. In order to achieve comprehension, one needs a process of learning about proper conversations and collaborations, but has to keep in mind that both meaning and understanding are relational. Therefore, Magritte's quote runs like a thread through the whole discussion. I would like to suggest further exploration of how knowledge visualization and mediated business analysis are related. Whilst the three-part series was aimed at establishing a basis for such an endeavor, it will have to take place in an applied setting. Knowledge visualization most definitely provides suitable opportunities and contexts.

\section{References}

[1] René Magritte. Magritte. Daniel Abadie and Patrick Roegiers, Eds. New York, D.A.P. 2003.

[2] Stefan Bertschi and Noah Bubenhofer. Linguistic Learning: A New Conceptual Focus in Knowledge Visualization. In Ninth International Conference on Information Visualisation (IV'05). London. July 2005. IEEE Computer Society Press. 383-389.

[3] Stefan Bertschi. Without Knowledge Visualization? Proposing a Deconstructivist Approach to Metaphor, Meaning and Perception. In Eleventh International Conference on Information Visualisation (IV'07). Zurich. July 2007. IEEE Computer Society Press. 342347.
[4] Remo Burkhard. Knowledge Visualization: The Use of Complementary Visual Representations for the Transfer of Knowledge - A Model, a Framework, and Four New Approaches. D.Sc. thesis. Swiss Federal Institute of Technology (ETH Zurich). 2005.

[5] Nick J. Enfield. The Theory of Cultural Logic: How Individuals Combine Social Intelligence with Semiotics to Create and Maintain Cultural Meaning. In Cultural Dynamics 12 (1). 2000. 35-64.

[6] Jochen Braun. Computational Neuroscience: Intimate Attention. In Nature 408 (6809). 2000. 154-155.

[7] Edgar Rubin. Figure and Ground. In Readings in Perception. David C. Beardslee and Michael Wertheimer, Eds. Princeton NJ, Van Nostrand. 1958. 194-203.

[8] Jason Maratos. The Power of Myth as Metaphor. In Group Analysis 39 (1). 2006. 87-99.

[9] Fathali M. Moghaddam. The Individual and Society: A Cultural Integration. New York, Worth Publishers. 2002.

[10] Warren Weaver. Recent Contributions to the Mathematical Theory of Communication. In The Mathematical Theory of Communication. Claude E. Shannon and Warren Weaver. Urbana, University of Illinois Press. 1964. 1-28.

[11] Josef Stern. Knowledge by Metaphor. In Midwest Studies in Philosophy 25. 2001. 187-226.

[12] Josef Stern. What Metaphors Do Not Mean. In Midwest Studies in Philosophy 16. 1991. 13-52.

[13] Raymond Hogler, Michael A. Gross, Jackie L. Hartman and Ann L. Cunliffe. Meaning in Organizational Communication: Why Metaphor Is the Cake, not the Icing. In Management Communication Quarterly 21 (3). 2008. 393-412.

[14] George Lakoff and Mark Johnson. Metaphors We Live By. Chicago, University of Chicago Press. 2003.

[15] Joep P. Cornelissen. Beyond Compare: Metaphor in Organization Theory. In Academy of Management Review 30 (4). 2005. 751-764.

[16] Richard H. Brown. Social Theory as Metaphor: On the Logic of Discovery for the Sciences of Conduct. In Theory and Society 3 (2). 1976. 169-197.

[17] International Institute of Business Analysis. Business Analysis Body of Knowledge (BABOK). Version 1.6 Final. Toronto, IIBA. 2008 [cf. Version 2.0. 2009].

[18] Thomas A. Schwandt. On Understanding Understanding. In Qualitative Inquiry 5 (4). 1999. 451-464.

[19] Mark Easterby-Smith, Richard Thorpe and Paul R. Jackson. Management Research. 3rd edition. London, Sage. 2008.

[20] Hubert Knoblauch. The Performance of Knowledge: Pointing and Knowledge in Powerpoint Presentations. In Cultural Sociology 2 (1). 2008. 75-97.

[21] Kristóf Nyíri. Wittgenstein's Philosophy of Pictures. In Wittgenstein Research Revisited. Conference at the HIT Centre. University of Bergen. December 2001. http://www.hunfi.hu/nyiri/nyiri bergen_tlk.htm.

[22] Erica Driver and Ron Rogowski. RIAs Bring PeopleCentered Design To Information Workplaces. Forrester Research. November 2007. 